\title{
Effect of acute kidney injury on mortality and hospital stay in patient with severe acute pancreatitis
}

\author{
JIAOJIAO ZHOU,${ }^{1}$ YI LI, ${ }^{2}$ YI TANG,${ }^{1}$ FANG LIU, ${ }^{1}$ SHAOBIN YU,${ }^{1}$ LING ZHANG, ${ }^{1}$ XIAOXI ZENG,${ }^{1}$ YULIANG $Z$ ZHAO $^{1}$ and \\ PING FU ${ }^{1}$ \\ ${ }^{1}$ Division of Nephrology, Kidney Research Institute, West China Hospital of Sichuan University, Chengdu, Sichuan, China; and ${ }^{2}$ Kidney Epidemiology and Cost \\ Center, Department of Biostatistics, University of Michigan, Ann Arbor, Michigan, USA
}

KEY WORDS:

Acute Kidney Injury Network, acute kidney injury, intensive care unit, risk factors, severe acute pancreatitis.

\section{Correspondence:}

Professor Ping Fu, Division of Nephrology, West China Hospital of Sichuan University, Chengdu 610041, China. Email: fupinghx@163.com

\section{Conflict of interest: None.}

Accepted for publication 22 February 2015 Accepted manuscript online 26 February 2015

doi:10.1111/nep.12439

\section{SUMMARY AT A GLANCE}

In this retrospective study of AKI following acute pancreatitis, the authors identify risk factors associated with mortality. These findings provide a basis for focussing on high risk patients for future trials of therapeutic interventions.

\begin{abstract}
Aim: Severe acute pancreatitis (SAP) is believed to be a major risk factor leading to acute kidney injury (AKI) among critically ill patients, but little is known about SAP-induced AKI. We study the incidence of AKI defined by the Acute Kidney Injury Network (AKIN) criteria and the risk factors associated with outcomes among SAP-induced AKI patients.

Method: We conducted a multicenter retrospective study of critically ill SAP-induced AKI patients during the period August 2009 to June 2013. Data on enrolled patients were retrieved from electronic records. Univariate and multiple regression analyses were performed.

Results: Among a total of 414 SAP patients admitted to intensive care units(ICU), $287(69.3 \%)$ developed AKI during their ICU stay, with 16.7\%, $18.4 \%$, and $34.3 \%$ classified as AKI stage I,II, and III, respectively. SAPinduced AKI patients experienced a significantly higher ICU mortality than those without AKI. The risk factors associated with ICU mortality among SAP-induced AKI patients included ACS (odds ratio (OR) 10.58), RRT (OR 3.31), sepsis (OR 2.46), CTSI (OR 3.01), APACHE II score (OR 1.82), AKI III (OR 1.38), ICU-length-of-stay (OR 1.04), and multi-organ failure.

Conclusions: The paper represents the first attempt to investigate the etiology and epidemiology of AKI following SAP under the AKIN criteria among critically ill patients. Several independent risk factors were found to be associated with ICU mortality for AKI patients. The findings may pinpoint crucial therapeutic measures for preventing AKI among a vulnerable population and for more effective management of SAP-induced AKI to improve the quality of intensive care.
\end{abstract}

Acute kidney injury (AKI) is any abrupt impairment (within $48 \mathrm{~h}$ ) of renal function and structure from any cause and is characterized by the rapid loss of the kidney's excretory function. It is typically diagnosed by the accumulation of end products of nitrogen metabolism (urea and creatinine) or decreased urine output, or both. Commonly associated with high morbidity and mortality, AKI is often secondary to extrarenal events and familiar multifactorial etiology. ${ }^{1,2}$ Severe acute pancreatitis (SAP), an acute abdominal disease with unpredictable clinical courses, is an important precursor of AKI and drastically increases the disease burden. ${ }^{3}$ For example, SAP leads to an incidence of AKI as high as 54\% among critically ill patients. ${ }^{4}$ Previous literature has also revealed a 10 -fold increase in mortality among critically ill SAP-induced AKI patients. ${ }^{5,6}$ However, SAP-induced AKI is greatly understudied, as the clinical characteristics and underlying processes of AKI coexisting with SAP are still elusive. Moreover, there are few clinical studies on etiologies 
and outcomes of SAP-induced AKI, especially among those receiving intensive care. Of particular interest is the fact that, even in the Kidney Disease: Improving Global Outcomes (KDIGO) summary reviewed by Kellum and Lameire, SAP was not mentioned as a cause of AKI. ${ }^{7}$

Since 2005, the Acute Kidney Injury Network (AKIN) has advocated several important changes in the AKI criteria. For example, the time constraint of diagnosis for AKI was introduced to ensure that the process was acute and representative of events within a clinically relevant time period, and the use of RRT was added as a criterion for AKI III. Some studies have indicated that the AKIN criteria could improve the sensitivity of AKI diagnosis. ${ }^{8-14}$ However, to our knowledge, only one study with 50 SAP-induced AKI patients applied the AKIN criteria. ${ }^{4}$

To address the knowledge gap, we plan to use the AKIN criteria to define and classify AKI following SAP onset and describe the clinical characteristics and laboratory results in intensive care. The paper describes the epidemiology of AKI with SAP under the AKIN criteria. Leveraging a relatively large cohort of critically ill patients, we aim to explore the severity of illness by using various scoring systems, investigate risk factors associated with the development of AKI, and describe the overall prognosis of this cohort.

\section{METHODS}

\section{Study protocol}

This was a retrospective and multi-center analysis of a database collected from a large cohort study of critically ill patients admitted to five ICU centers due to SAP in the time period between August 2009 to June 2013. Requirement for approval by the local ethics committee and the need for informed consent were waived because the retrospective study required neither therapeutic or prophylactic intervention nor contravention of privacy or confidentiality.

\section{Study population}

All patients who were over 18 years old and admitted to the ICU with evidence of SAP were eligible. However, patients who were treated with renal replacement therapy (RRT) within the past month or were on dialysis at ICU admission and had received kidney transplant were excluded.

\section{Data collection}

Patients' age, sex, laboratory data, clinical details, including the aetiology of SAP, AKIN classification, disease history, presence of comorbidity, and partial treatment, were collected in the database. The primary outcomes were documented as the ICU- length-of-stay (ICU-LOS) and mortality in ICU. The predictive role of two general severity of illness scoring systems (Acute Physiology and Chronic Health Evaluation (APACHE) II and Sequential Organ Failure Assessment (SOFA) scores) and two disease-specific scoring systems (Computed Tomography Severity Index (CTSI) and Ranson scores) were calculated using the worst values obtained in the first $48 \mathrm{~h}$ after admission to ICU. Partial treatments such as operations, RRT, mechanical ventilation, vasopressor use and diuretics were considered. The highest number of failed organs involved (except kidney) during the ICU stay was summarized and recorded as 'maximum number of associated failed organ systems' for each patient.

\section{Definition}

In our study, AKI is defined as an absolute increase in serum creatinine of greater than or equal to $0.3 \mathrm{mg} / \mathrm{dL}(\geq 26.4 \mu \mathrm{mol} / \mathrm{L})$ and a percentage increase in serum creatinine of greater than or equal to $50 \%$ (1.5-fold from baseline) within $48 \mathrm{~h}$. We define the lowest value of serum creatinine measured within 2 days prior to ICU admission as the baseline creatinine value. If no serum creatinine was available, we considered the first creatinine available within 2 days after ICU admission as the baseline serum creatinine values. AKI is classified as AKI stage I, II, or III, according to the patient's level of serum creatinine (Table 1). As 6- and 12-hourly urine volumes were largely missing in the database, we used creatinine to define and classify AKI and compare it with subsequent values every 2 days until death or discharge from ICU. The highest AKI stage during patients' ICU stay defines the final AKI classification. SAP, as proposed by Bradley, known as the Atlanta classification, is established by either an APACHE II score $>8$, Ranson score $>3$, the presence of more than one organ failure, or local complications. ${ }^{15}$ Sepsis was defined as the presence of positive blood cultures accompanied by systemic inflammatory response syndrome (SIRS). ${ }^{16,17}$ The World Society for Abdominal Compartment Syndrome (WSACS), an international multi-specialty consortium proposed that intraabdominal pressure (IAP) is defined as sustained or repeated pathologic elevation of IAP $\geq 12 \mathrm{mmHg}$ and ACS is defined as a sustained IAP $\geq 20 \mathrm{mmHg}$ that is associated with new organ dysfunction. ${ }^{18}$ The scoring system of CTSI, modified by Mortele et al. in 2004, includes pancreatic inflammation, pancreatic necrosis and extrapancreatic complications. ${ }^{19}$ At 48 h post-admission, a Ranson score of $\geq 3$ defines severe pancreatitis. ${ }^{20}$ Organ system failures were assessed according to the method proposed by Knaus and colleagues, supplemented by a definition for gastrointestinal failure (failure to tolerate enteral nutrition)..$^{21,22}$

Table 1 Classification system for acute kidney injury (AKI)

\begin{tabular}{|c|c|c|}
\hline $\begin{array}{l}\text { AKIN } \\
\text { stage }\end{array}$ & Serum creatinine criteria & Urine output criteria \\
\hline 1 & $\begin{array}{l}\text { Increase in serum creatinine of more than } \\
\text { or equal to } 0.3 \mathrm{mg} / \mathrm{dL} \text { ( } \geq 26.4 \mu \mathrm{mol} / \mathrm{L} \text { ) or } \\
\text { increase to more than or equal to } 150 \% \\
\text { to } 200 \% \text { (1.5- to } 2 \text {-fold) from baseline }\end{array}$ & $\begin{array}{l}\text { Less than } 0.5 \mathrm{~mL} / \mathrm{kg} \\
\text { per hour for more } \\
\text { than } 6 \mathrm{~h}\end{array}$ \\
\hline 2 & $\begin{array}{l}\text { Increase in serum creatinine to more } \\
\text { than } 200 \% \text { to } 300 \% \text { (>2- to 3-fold) from } \\
\text { baseline }\end{array}$ & $\begin{array}{l}\text { Less than } 0.5 \mathrm{~mL} / \mathrm{kg} \\
\text { per hour for more } \\
\text { than } 12 \mathrm{~h}\end{array}$ \\
\hline 3 & $\begin{array}{l}\text { Increase in serum creatinine to more } \\
\text { than } 300 \% \text { (>3-fold) from baseline (or } \\
\text { serum creatinine of more than or equal } \\
\text { to } 4.0 \mathrm{mg} / \mathrm{dL} \text { ( } \geq 354 \mu \mathrm{mol} / \mathrm{L} \text { ) with an } \\
\text { acute increase of at least } 0.5 \mathrm{mg} / \mathrm{dL} \\
\text { (44 } \mu \mathrm{mol} / \mathrm{L} \text { )) }\end{array}$ & $\begin{array}{l}\text { Less than } 0.3 \mathrm{~mL} / \mathrm{kg} \\
\text { per hour for } 24 \mathrm{~h} \text { or } \\
\text { anuria for } 12 \mathrm{~h}\end{array}$ \\
\hline
\end{tabular}




\section{Statistical analysis}

In our descriptive analysis, continuous variables are described as mean \pm standard deviations (SD) and 95\% confidence intervals (CI). Categorical variables are presented as proportions. Student's t-tests, one-way ANOVA and Pearson $\chi^{2}$ tests are used in the univariate analysis to evaluate statistical associations. Multiple logistic regression is utilized to identify independent predictors of ICU mortality, based on which odds ratios (ORs) are calculated. Risk factors that are found to be significant in univariate analyses are considered in the multiple regression model. A two-sided $P$-value $<0.05$ is considered statistically significant. The analysis is conducted using SPSS 17.0 (SPSS, Chicago, IL, USA).

\section{RESULTS}

A total of 414 patients with confirmed diagnosis of SAP during the period of August 2009 to June 2013 are included in our analysis. There are $287(69.3 \%)$ SAP-induced AKI patients, among whom $24.0 \%, 26.5 \%$ and $49.5 \%$ are classified as AKI I, II, and III, respectively, according to the AKIN criteria. The mean $( \pm S D)$ age is 50.2 (15.2) years, $56.8 \%$ of patients are male, and ICU-LOS is 22.6 (10.6) days. Overall ICU mortality is $37.4 \%(155 / 414)$ and AKI is associated with a significantly higher ICU mortality (44.9\% versus $20.5 \%$, $P<0.001)$ when compared to SAP patients without AKI. The major causes of SAP are gallstone disease $(40.3 \%)$ and alcohol abuse $(38.9 \%)$. The primary disease history and laboratory data of patients who entered the ICU are summarized in Table 2. The principal systemic complications are SIRS $(54.1 \%)$, followed by ACS (53.4\%), and acute lung injury (ALI) $(44.9 \%)$. The mean $( \pm \mathrm{SD})$ APACHE II score is 17.0 (7.9); the SOFA score, 9.4 (5.1); the Ranson score, 6.2 (1.7); and CTSI, $6.2(2.5)$. The mean $( \pm S D)$ baseline serum creatinine level was 365.2 (284.7) $\mu \mathrm{mol} / \mathrm{L}$. Other laboratory data and partial treatment are also presented in Table 2. Table 3 shows an increasing linear trend with percentages of $29.0 \%$ for AKI I, 39.5\% for AKI II, and 56.6\% for AKI III. Figure 1 depicts serum creatinine level trend in non-AKI and AKI groups during their ICU stay.

A comparison of the basic characteristics and laboratory variables between SAP patients with and without AKI are listed in Table 3. The proportions of chronic kidney disease (CKD), sepsis, and ACS are significantly higher in the AKI group than in the non-AKI group $(P<0.05)$. A number of factors are associated with significant differences between AKI I, II, and III patients in Table $3(P<0.05)$. Patients with AKI are mostly older and have higher baseline serum creatinine, blood urea nitrogen (BUN), Cystain-C, and C-reactive protein (CRP) levels when compared with patients without AKI $(P<0.05)$. However, there was no significant difference between having AKI and not having AKI in the aetiology, and also no significant difference among AKI I, II, and III patients. Meanwhile, ICU-LOS was not a significant risk factor and did not distinguish patients who developed AKI and those who did not (Table 3).
Table 2 Baseline characteristics of study population

\begin{tabular}{|c|c|}
\hline Age (years; mean \pm SD) & $50.2 \pm 15.2$ \\
\hline \multicolumn{2}{|l|}{ Gender, $n(\%)$} \\
\hline Male & $235(56.8)$ \\
\hline Female & $179(43.2)$ \\
\hline \multicolumn{2}{|l|}{ AKI stage, $n$ (\%) } \\
\hline No-AKI & $127(30.7)$ \\
\hline AKII & $69(16.7)$ \\
\hline AKI II & $76(18.4)$ \\
\hline AKI III & $142(34.3)$ \\
\hline ICU-LOS (days; mean \pm SD) & $22.6 \pm 10.6$ \\
\hline \multicolumn{2}{|l|}{ Aetiology, $n(\%)$} \\
\hline Biliary & $167(40.3)$ \\
\hline Alcohol & $161(38.9)$ \\
\hline Other & $86(20.8)$ \\
\hline \multicolumn{2}{|l|}{ Disease history, $n$ (\%) } \\
\hline Hypertension & $94(22.7)$ \\
\hline Diabetes & $48(11.6)$ \\
\hline Heart failure & $80(19.3)$ \\
\hline Chronic liver disease & $62(15.0)$ \\
\hline Malignancy & $15(3.6)$ \\
\hline COPD & $83(20.0)$ \\
\hline CKD & $38(9.2)$ \\
\hline \multicolumn{2}{|l|}{ Comorbidity, $n \quad(\%)$} \\
\hline $\mathrm{ALI}$ & $186(44.9)$ \\
\hline ARDS & $164(39.6)$ \\
\hline ALF & $22(5.3)$ \\
\hline SIRS & $224(54.1)$ \\
\hline Sepsis & $90(21.7)$ \\
\hline Hypoalbuminemia & $138(33.3)$ \\
\hline ACS & $221(53.4)$ \\
\hline \multicolumn{2}{|l|}{ Laboratory data, mean \pm SD } \\
\hline HGB $(g / L)$ & $89.1 \pm 16.3$ \\
\hline Platelets $\left(10^{9} / \mathrm{L}\right)$ & $160.0 \pm 79.7$ \\
\hline WBC $\left(10^{9} / \mathrm{L}\right)$ & $14.3 \pm 6.6$ \\
\hline Baseline serum creatinine $(\mu \mathrm{mol} / \mathrm{L})$ & $365.2 \pm 284.7$ \\
\hline BUN (mmol/L) & $13.00 \pm 5.8$ \\
\hline UA $(\mu \mathrm{mol} / \mathrm{L})$ & $421.4 \pm 141.6$ \\
\hline Cystain-C (mg/L) & $1.8 \pm 0.6$ \\
\hline AST (IU/L) & $467.2 \pm 325.2$ \\
\hline ALT (IU/L) & $497.2 \pm 304.1$ \\
\hline $\mathrm{Lac}(\mathrm{mmol} / \mathrm{L})$ & $5.7 \pm 2.6$ \\
\hline $\mathrm{LDH}(\mathrm{IU} / \mathrm{L})$ & $1083.8 \pm 980.9$ \\
\hline Glucose (mmol/L) & $14.0 \pm 5.5$ \\
\hline AMY (IU/L) & $1024.80 \pm 742.3$ \\
\hline LPS (IU/L) & $1163.28 \pm 881.0$ \\
\hline $\mathrm{Ca}(\mathrm{mmol} / \mathrm{L})$ & $1.7 \pm 0.4$ \\
\hline CRP (mg/L) & $175.08 \pm 74.19$ \\
\hline \multicolumn{2}{|l|}{ Scoring systems, mean \pm SD } \\
\hline APACHE II score & $17.00 \pm 7.9$ \\
\hline SOFA score & $9.4 \pm 5.1$ \\
\hline Ranson score & $6.2 \pm 1.7$ \\
\hline CTSI & $6.2 \pm 2.5$ \\
\hline \multicolumn{2}{|l|}{ Therapy requirements, $n$ (\%) } \\
\hline Operation & $88(21.3)$ \\
\hline RRT & $245(59.2)$ \\
\hline Mechanical Ventilation & 28568.8) \\
\hline Vasopressor use & $157(37.9)$ \\
\hline Diuretic & $120(29.0)$ \\
\hline
\end{tabular}

ACS, abdominal compartment syndrome; AKI, acute kidney injury; ALF, acute liver failure; ALI, acute lung injury; ALT, aspartate transaminase; AMY, amylase; APACHE II, Acute Physiology and Chronic Health Evaluation scoring system version II; ARDS, acute respiratory distress syndrome; AST, aspartate aminotransferase; BUN, blood urea nitrogen; CKD, chronic kidney disease; COPD, chronic obstructive pulmonary disease; CRP, C-reactive protein; CTSI, Computed Tomography Severity Index; HGB, hemoglobin; ICU-LOS, intensive care unit length of stay; Lac, lactic acid; LDH, lactic dehydrogenase; LPS, lipase; RRT, renal replacement therapy; SIRS, systemic inflammatory response syndrome; SOFA, Sequential Organ Failure Assessment; UA, uric acid; WBC, white blood cell. 
Table 3 Clinical characteristics and laboratory data associated with acute kidney injury (AKI) and Non-AKI

\begin{tabular}{|c|c|c|c|c|c|c|}
\hline & $\begin{array}{l}\text { Non-AKI } \\
n=127\end{array}$ & $\begin{array}{l}\text { AKI I } \\
n=69\end{array}$ & $\begin{array}{l}\text { AKI II } \\
n=76\end{array}$ & $\begin{array}{c}\text { AKI III } \\
n=142\end{array}$ & $\begin{array}{c}P_{1} \\
\text { AKI vs Non-AKI }\end{array}$ & $\begin{array}{c}P_{2} \\
\text { Among AKI I, II, III }\end{array}$ \\
\hline Age, years & $46.6 \pm 14.8$ & $53.2 \pm 16.1$ & $46.4 \pm 14.2$ & $48.8 \pm 15.0$ & 0.021 & 0.014 \\
\hline Gender (Female), $n(\%)$ & $50(39.4)$ & 51 (73.9) & $31(40.8)$ & $47(33.1)$ & 0.177 & 0.031 \\
\hline Mortality, $n(\%)$ & $26(20.5)$ & $20(29.0)$ & $30(39.5)$ & 79 (55.6) & $<0.001$ & $<0.001$ \\
\hline \multicolumn{7}{|l|}{ Aetiology, $n(\%)$} \\
\hline Biliary & $57(44.9)$ & 37 (53.6) & $24(31.6)$ & 49 (34.5) & 0.210 & 0.362 \\
\hline Alcohol & $48(37.8)$ & $34(49.3)$ & $34(44.7)$ & $45(31.7)$ & 0.761 & 0.526 \\
\hline Others & $22(17.3)$ & $18(26.1)$ & $18(23.7)$ & $28(19.4)$ & 0.250 & 0.846 \\
\hline \multicolumn{7}{|l|}{ Disease history, n (\%) } \\
\hline Hypertension & $31(24.4)$ & $23(33.3)$ & $11(14.5)$ & $29(20.4)$ & 0.582 & 0.174 \\
\hline Diabetes & $14(11.0)$ & $15(21.7)$ & $6(7.9)$ & $13(9.2)$ & 0.809 & 0.179 \\
\hline Heart failure & $38(29.9)$ & $18(26.1)$ & $11(14.5)$ & $13(9.2)$ & $<0.001$ & $<0.001$ \\
\hline Chronic liver disease & $18(14.2)$ & $13(18.8)$ & $6(7.9)$ & $25(17.6)$ & 0.761 & 0.056 \\
\hline Malignancy & $3(2.4)$ & $5(7.2)$ & $3(3.9)$ & $4(2.8)$ & 0.361 & 0.702 \\
\hline COPD & $25(19.7)$ & $25(36.2)$ & 9 (11.8) & $24(16.9)$ & 0.902 & 0.034 \\
\hline CKD & $6(4.7)$ & $6(8.7)$ & $8(10.5)$ & $18(12.7)$ & 0.037 & 0.158 \\
\hline Sepsis & $22(17.3)$ & $23(33.3)$ & $21(27.6)$ & $24(16.9)$ & $<0.001$ & 0.362 \\
\hline ACS & $62(48.8)$ & $42(60.1)$ & 39 (51.3) & $78(54.9)$ & $<0.001$ & $<0.001$ \\
\hline ICU-LOS, days & $22.4 \pm 10.3$ & $24.8 \pm 15.8$ & $22.6 \pm 11.4$ & $25.0 \pm 12.5$ & 0.074 & 0.218 \\
\hline \multicolumn{7}{|l|}{ Laboratory data, mean \pm SD } \\
\hline HGB (g/L) & $89.9 \pm 16.2$ & $90.7 \pm 16.7$ & $87.3 \pm 16.3$ & $88.1 \pm 15.9$ & 0.445 & 0.360 \\
\hline PLT (109/L) & $165.6 \pm 81.2$ & $167.8 \pm 79.9$ & $156.2 \pm 85.6$ & $150.9 \pm 73.9$ & 0.346 & 0.303 \\
\hline WBC $\left(10^{9} / \mathrm{L}\right)$ & $14.0 \pm 6.4$ & $14.3 \pm 7.4$ & $13.5 \pm 5.8$ & $15.2 \pm 6.7$ & 0.487 & 0.229 \\
\hline Baseline serum creatinine $(\mu \mathrm{mol} / \mathrm{L})$ & $119.4 \pm 95.7$ & $185.9 \pm 142.7$ & $393.3 \pm 198.9$ & $734.5 \pm 386.3$ & $<0.001$ & $<0.001$ \\
\hline BUN (mmol/L) & $11.5 \pm 4.8$ & $13.6 \pm 6.3$ & $12.8 \pm 5.5$ & $14.2 \pm 6.4$ & $<0.001$ & 0.288 \\
\hline UA ( $\mu \mathrm{mol} / \mathrm{L})$ & $415.8 \pm 124.8$ & $406.1 \pm 143.1$ & $445.0 \pm 123.5$ & $423.8 \pm 165.2$ & 0.565 & 0.245 \\
\hline Cystain-C (mg/L) & $1.6 \pm 0.5$ & $1.7 \pm 0.5$ & $1.8 \pm 0.6$ & $2.0 \pm 0.6$ & $<0.001$ & 0.007 \\
\hline AST (IU/L) & $449.4 \pm 339.8$ & $540.7 \pm 420.8$ & $719.0 \pm 412.8$ & $629.3 \pm 415.0$ & $<0.001$ & $<0.001$ \\
\hline ALT (IU/L) & $465.2 \pm 341.1$ & $427.7 \pm 306.1$ & $716.5 \pm 488.6$ & $759.3 \pm 403.1$ & $<0.001$ & $<0.001$ \\
\hline Lac (mmol/L) & $5.9 \pm 2.5$ & $5.6 \pm 3.0$ & $5.1 \pm 2.4$ & $5.9 \pm 2.5$ & 0.361 & 0.163 \\
\hline $\mathrm{LDH}(\mathrm{IU} / \mathrm{L})$ & $983.1 \pm 674.6$ & $1160.2 \pm 720.5$ & $1027.0 \pm 597.5$ & $1168.2 \pm 641.5$ & 0.165 & 0.639 \\
\hline Glucose (mmol/L) & $13.8 \pm 5.1$ & $13.8 \pm 5.8$ & $11.1 \pm 6.0$ & $14.5 \pm 5.5$ & 0.485 & 0.641 \\
\hline AMY (IU/L) & $877.6 \pm 488.7$ & $1052.1 \pm 875.0$ & $1112.1 \pm 787.8$ & $1118.5 \pm 922.1$ & 0.033 & 0.059 \\
\hline LPS (IU/L) & $1053.4 \pm 865.4$ & $1108.6 \pm 1580.8$ & $1262.6 \pm 733.0$ & $1392.3 \pm 865.5$ & 0.042 & 0.063 \\
\hline $\mathrm{Ca}(\mathrm{mmol} / \mathrm{L})$ & $1.8 \pm 0.4$ & $1.6 \pm 0.4$ & $1.8 \pm 0.4$ & $1.7 \pm 0.4$ & 0.277 & 0.046 \\
\hline CRP (mg/L) & $192.9 \pm 82.3$ & $177.4 \pm 82.1$ & $146.7 \pm 55.6$ & $172.5 \pm 63.8$ & 0.002 & 0.009 \\
\hline
\end{tabular}

ACS, abdominal compartment syndrome; ALT, aspartate transaminase; AMY, amylase; AST, aspartate aminotransferase; BUN, blood urea nitrogen; CKD, chronic kidney disease; COPD, chronic obstructive pulmonary disease; CRP, C-reactive protein; HGB, hemoglobin; Lac, lactic acid; LDH, lactic dehydrogenase; LPS, lipase; UA, uric acid; WBC, white blood cell.

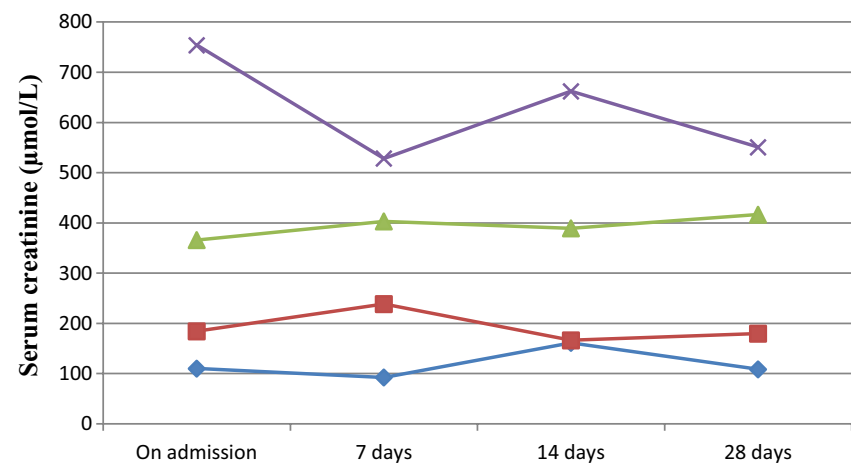

Fig. 1 The trend of renal function change in non-acute kidney injury (AKI) and AKI groups. $(-)$ Non-AKI, $(-)$ AKI I, $(-)$ AKI II, $(*)$ AKI III.
We next performed a multiple logistic regression on these 287 SAP-induced AKI patients to identify the effect of the independent risk factors on ICU mortality. Our univariate analysis identified that ICU-LOS, AKIN stage, SIRS, sepsis, hypoproteinaemia, ACS, ARDS, ALI, and maximum number of associated organ failures were significant risk factors. The APACHE II score and CTSI are higher in non-survivors (Table 4). However, there are no significant differences in age, CKD, Ranson and SOFA score $(P>0.05)$ between both groups. The results of multivariate logistic regression analysis are shown in Table 5. After adjustment for covariates, in SAP patients with AKI, independent risk factors for mortality included ACS (OR 10.58; 95\% CI 5.98 to 18.72), renal replacement therapy (RRT) (OR 3.31; 95\%CI 1.77 to 6.19), sepsis (OR 2.46; $95 \%$ CI 1.10 to 4.01 ), Computed 
Table 4 Univariate analysis of risk on intensive care unit (ICU) mortality for patients with acute kidney injury (AKI) following severe acute pancreatitis (SAP)

\begin{tabular}{|c|c|c|c|}
\hline Variables & $\begin{array}{l}\text { Non-survivors } \\
\qquad(n=129)\end{array}$ & $\begin{array}{l}\text { Survivors } \\
(n=158)\end{array}$ & $P$-value \\
\hline Age, year & $51.2 \pm 15.2$ & $48.4 \pm 15.0$ & 0.116 \\
\hline Gender (Female), $n(\%)$ & $54(41.9)$ & $71(44.9)$ & 0.601 \\
\hline ICU-LOS, days & $25.9 \pm 15.7$ & $20.8 \pm 9.2$ & 0.001 \\
\hline \multicolumn{4}{|l|}{ AKI stage, $n(\%)$} \\
\hline AKII & $20(15.5)$ & 49 (31.0) & $<0.001$ \\
\hline AKI II & $30(23.3)$ & $46(29.1)$ & $<0.001$ \\
\hline AKI III & $79(61.2)$ & $63(39.9)$ & $<0.001$ \\
\hline \multicolumn{4}{|l|}{ The etiology, $n(\%)$} \\
\hline Biliary & $48(37.2)$ & $62(39.2)$ & 0.653 \\
\hline Alcohol & $49(38.0)$ & $64(40.5)$ & 0.436 \\
\hline Other & $32(24.8)$ & $32(20.3)$ & 0.261 \\
\hline \multicolumn{4}{|l|}{ Disease history, $n(\%)$} \\
\hline Hypertension & $33(25.6)$ & $30(19.0)$ & 0.179 \\
\hline Diabetes & $17(13.2)$ & $17(10.8)$ & 0.528 \\
\hline Heart failure & $22(17.1)$ & $24(15.2)$ & 0.668 \\
\hline Chronic liver disease & 19 (14.7) & $25(15.8)$ & 0.798 \\
\hline Malignancy & $5(3.9)$ & $7(4.4)$ & 0.815 \\
\hline COPD & $30(23.3)$ & $28(17.7)$ & 0.245 \\
\hline CKD & $12(9.3)$ & $20(12.7)$ & 0.369 \\
\hline \multicolumn{4}{|l|}{ Comorbidity, $n(\%)$} \\
\hline $\mathrm{ALI}$ & $58(45.0)$ & $51(32.3)$ & 0.028 \\
\hline ARDS & $68(52.7)$ & 59 (37.3) & 0.009 \\
\hline ALF & $10(7.8)$ & $8(5.1)$ & 0.350 \\
\hline SIRS & $76(58.9)$ & $68(43.0)$ & 0.007 \\
\hline Sepsis & $33(25.6)$ & $25(15.8)$ & 0.031 \\
\hline Hypoproteinemia & $53(41.1)$ & $39(24.7)$ & 0.003 \\
\hline ACS & $88(68.2)$ & 71 (44.9) & 0.012 \\
\hline \multicolumn{4}{|c|}{ Scoring systems, mean \pm SD } \\
\hline APACHE II score & $18.1 \pm 8.7$ & $15.2 \pm 6.6$ & $<0.001$ \\
\hline SOFA score & $9.8 \pm 5.2$ & $8.8 \pm 4.9$ & 0.089 \\
\hline Ranson score & $6.3 \pm 1.8$ & $6.1 \pm 1.9$ & 0.067 \\
\hline CTSI & $6.5 \pm 2.7$ & $5.7 \pm 1.9$ & 0.007 \\
\hline \multicolumn{4}{|c|}{$\begin{array}{l}\text { Maximum number of associated failed organ systems during ICU stay } \\
\text { (excluding AKI) }\end{array}$} \\
\hline 1 failed organ & 21 & 23 & $<0.001$ \\
\hline 2 failed organs & 36 & 43 & $<0.001$ \\
\hline 3 failed organs & 82 & 48 & $<0.001$ \\
\hline$\geq 4$ failed organs & 24 & 10 & $<0.001$ \\
\hline \multicolumn{4}{|c|}{ Therapy requirements, $n(\%)$} \\
\hline Operation & $29(22.5)$ & $22(13.9)$ & 0.059 \\
\hline RRT & $89(69.0)$ & $64(40.5)$ & 0.011 \\
\hline Mechanical ventilation & $106(82.2)$ & $77(48.7)$ & 0.004 \\
\hline Vasopressor use & $51(39.5)$ & $50(31.6)$ & 0.164 \\
\hline Diuretic & $30(23.3)$ & $47(29.4)$ & 0.362 \\
\hline
\end{tabular}

ACS, abdominal compartment syndrome; ALF, acute liver failure; ALI, acute lung injury; APACHE II, Acute Physiology and Chronic Health Evaluation scoring system version II; ARDS, acute respiratory distress syndrome; CKD, chronic kidney disease; COPD, chronic obstructive pulmonary disease; CTSI, computed tomography Severity Index; RRT, renal replacement therapy; SIRS, systemic inflammatory response syndrome; SOFA, Sequential Organ Failure Assessment.
Table 5 Risk factors for intensive care unit (ICU) mortality according to multivariate logistic regression analysis

\begin{tabular}{lccccr}
\hline & $\beta$ & $P$ & OR & \multicolumn{2}{c}{$\begin{array}{c}\text { 95\% confidence } \\
\text { interval }\end{array}$} \\
\cline { 5 - 6 } & & & & Lower & Upper \\
\hline ICU-LOS & 0.04 & 0.03 & 1.04 & 1.01 & 1.07 \\
AKI III & 0.97 & 0.01 & 1.38 & 1.19 & 1.77 \\
Sepsis & 0.74 & 0.21 & 2.46 & 1.10 & 4.01 \\
ACS & 2.36 & $<0.001$ & 10.58 & 5.98 & 18.72 \\
CTSI & 2.14 & 0.01 & 3.01 & 1.78 & 5.32 \\
APACHE II score & 1.35 & 0.04 & 1.82 & 1.23 & 2.72 \\
Maximum number of associated failed organ systems during ICU stay & \\
$\quad$ (excluding AKI) & & & & & 1.09 \\
2 & 1.48 & $<0.001$ & 1.23 & 1.55 \\
3 & 1.04 & 0.01 & 1.75 & 1.45 & 2.63 \\
$\geq 4$ & 1.01 & 0.04 & 2.36 & 2.14 & 2.93 \\
RRT & 1.20 & $<0.001$ & 3.31 & 1.77 & 6.19 \\
\hline
\end{tabular}

AKI, acute kidney injury; ACS, Abdominal Compartment Syndrome; APACHE II, Acute Physiology and Chronic Health Evaluation scoring system version II; CTSI, computed tomography Severity Index; ICU-LOS, intensive care unitlength-of-stay RRT, renal replacement therapy.

Tomography Severity Index (CTSI) (OR 3.01; 95\% CI 1.78 to 5.32), APACHE II score (OR 1.82; $95 \%$ CI 1.23 to 2.72), AKI III (OR 1.38; 95\% CI 1.19 to 1.77), ICU-LOS (OR 1.04; 95\% CI 1.01 to 1.07 ), and multi-organ failure (Table 5).

\section{DISCUSSION}

Despite significant progress in the management of patient treatment, such as early enteral nutrition, decompression surgery, and RRT, SAP remains a severe medical condition with multi-organ failure and a poor clinical prognosis, especially in critically ill patients. ${ }^{23}$ We conducted a retrospective analysis of ICU patients, validated the ability of the AKIN criteria to evaluate the occurrence of AKI in SAP patients, and assessed risk factors for ICU mortality.

Our results suggest that AKI patients experience a significantly higher ICU mortality compared with those who never develop AKI during intensive care $(20.5 \%$ vs $44.9 \%$, $P<0.001$ ), which is consistent with published reports. ${ }^{24,25}$ The mortality in the AKI III group is the highest at 55.6\% compared with the AKI I and II groups. Moreover, more advanced AKIN classifications are associated with higher rates of ICU mortality, in an approximately linear fashion. Our analysis demonstrates that AKI is more common among older patients and the incidence of ACS increases as the AKI stage increases (Table 3). In our study, heart failure rate and CRP level were higher in the non-AKI group than in the AKI group. Both results were surprising, compared to many other reports on AKI. Possible reasons include differences in the populations characteristics studied, and different criteria in the diagnosis of AKI, for example, RIFLE criteria or KDIGO criteria. In addition, SAP is a dysregulated inflammatory 
response to infection and it can lead to multiple-organ dysfunction syndrome in the end. The CRP level cannot completely reflect systemic inflammation in critically ill patients.

Our analysis has identified several factors as predictors for ICU mortality. ICU-LOS, AKI III (using AKI I as the reference classification), sepsis, ACS, CTSI, APACHE II score, RRT, and multiple failed organ systems (using one failed organ system as the reference classification) are the primary and independent factors for mortality in this cohort. Patients with a history of renal disease who develop SAP have a significant risk factor for developing AKI, but it is not an independent prognostic factor, in contradiction with some studies. ${ }^{5,24,26}$ Meanwhile, our conclusion that the type of treatment, except for RRT, is not independently associated with ICU mortality also contrasts with studies that focused on other types of nephrology. ${ }^{12,27-29}$

Several studies have asserted that renal failure is always one of the main drivers in multi-organ failure due to SAP. ${ }^{23,30}$ The main pathophysiology is initiated as an uncontrolled systemic inflammatory process with ensuing hypovolaemia, either from wide-spread vasodilation or fluid sequestration, and reduced tissue perfusion, which ultimately causes organ damage and failure, even multi-organ failure. ${ }^{24}$ We show that multi-organ failure is an important prognostic factor in SAP induced-AKI as reported previously. ${ }^{5,31,32}$

Sepsis is a highly prevalent syndrome characterized by a whole-body inflammatory state and the presence of a known or suspected infection that has severe consequences, including multi-organ failure. ${ }^{10}$ Previous reports have suggested that AKI is frequently considered to be a serious complication of sepsis in ICU patients and that septic AKI effects an important and independent increase in the risk for hospital death. ${ }^{10,27,33}$ In our study, the prevalence of sepsis as a complication of SAP was $21.7 \%$. AKI occurred in $75.6 \%$ (68/90) of all critically ill patients with both SAP and sepsis. In multivariate logistic regression analysis, the sepsis showed an ICU mortality odds ratio of $2.46(P<0.001)$. The findings of our study confirm and largely extend a previous investigation. ${ }^{34}$

There are several different scoring systems aimed at estimating the prognosis of patients with SAP. ${ }^{20,35-38}$ The APACHE II and the SOFA scores are general severity of illness scoring systems that are positively correlated with SAP severity and have good prognostic value. ${ }^{35,37,39}$ The Ranson scores are the most commonly used and the CTSI has been shown to have good predictive value. ${ }^{20,38}$ However, in our original study, we find only that CTSI and APACHE II scores were significant risk factors for AKI in patients with SAP, at variance with previous studies. An adequately powered validation study is necessary to evaluate the role of the various scores in the AKI cohort following SAP onset.

\section{Limitations}

There are, however, some limitations in our study. First, the diagnosis of SAP was made partly at the discretion of indi- vidual investigators. Second, due to lack of exact and complete urine volumes, we had to resort to serum creatinine levels obtained in the relevant period. As a result, we may have underestimated patients who only fulfilled the AKI criteria on urine output and, perhaps, underestimated the incidence of AKI. Third, there is no cause-effect relationship between ICU stay and development of AKI that can be drawn in this cross-sectional study, but we have verified risk factors that may be associated with AKI in the hospital or ICU settings as in previous research. Finally, as no data on long-term follow-up were available beyond the ICU discharge, patients' long-term mortality and renal conditions remain unknown.

\section{Conclusion}

Few epidemiological studies have been conducted on the SAP-induced AKI patients cohorts. The results of the present research suggest that the AKIN criteria might offer sensitive diagnostic measures among SAP patients. We have found that ACS, RRT, sepsis, CTSI, APACHE II score, AKI III, ICULOS, and multi-organ failure are significant risk factors associated with high ICU mortality among the SAP-induced AKI patients. Our findings have the potential to lead to effective therapeutic measures to prevent and manage SAP-induced AKI, with the ultimate goal of improving survival outcomes among critically ill patients.

\section{ACKNOWLEDGEMENTS}

This work was partially supported by Grant-in-aid for scientific research from the National Natural Science Foundation of China (No. 81270818). We thank Kangyi Zhang, Xiaofeng Su and, Xiaoyan Zhang for data collection, and thank Yong Jiang for his help in statistical analyses. We are grateful to the ICU in the West China Hospital of Sichuan University for facilitating our study. We thank Ruth Shamraj for proofreading this article and for many useful editorial suggestions.

\section{REFERENCES}

1. Bellomo R, Kellum JA, Ronco C. Acute kidney injury. Lancet 2012; 380: 756-66.

2. Chertow GM, Burdick E, Honour M, Bonventre JV, Bates DW. Acute kidney injury, mortality, length of stay, and costs in hospitalized patients. J. Am. Soc. Nephrol. 2005; 16: 3365-70.

3. Petejova N, Martinek A. Acute kidney injury following acute pancreatitis: A review. Biomed. Pap. Med. Fac. Univ. Palacky Olomouc Czech. Repub 2013; 157: 105-13.

4. Pavlidis $\mathrm{P}$, Crichton S, Lemmich Smith J et al. Improved outcome of severe acute pancreatitis in the intensive care unit. Crit. Care Res. Pract. 2013; 2013: 897107.

5. Kes P, Vucicevic Z, Ratkovic-Gusic I, Fotivec A. Acute renal failure complicating severe acute pancreatitis. Ren. Fail. 1996; 18: 621-8.

6. Herrera Gutierrez ME, Seller Perez G, de La Rubia De Gracia C, Chaparro Sanchez MJ, Nacle Lopez B. Acute renal failure profile 
and prognostic value in severe acute pancreatitis. Med. Clin. (Barc) 2000; 115: 721-5.

7. Kellum JA, Lameire N. Diagnosis, evaluation, and management of acute kidney injury: A KDIGO summary (Part 1). Crit. Care 2013; 17: 204.

8. Kwon SH, Noh H, Jeon JS, Kim Y, Han DC. An assessment of AKIN criteria for hospital-acquired acute kidney injury: A prospective observational cohort study. Nephron Clin. Pract. 2010; 116: c217-23.

9. Lopes JA, Fernandes P, Jorge S et al. Acute kidney injury in intensive care unit patients: A comparison between the RIFLE and the Acute Kidney Injury Network classifications. Crit. Care 2008; 12: R110.

10. Zarjou A, Agarwal A. Sepsis and acute kidney injury. Journal of the American Society of Nephrology. J. Am. Soc. Nephrol. 2011; 22: 999-1006.

11. De Waele JJ, De Laet I, Kirkpatrick AW, Hoste E. Intra-abdominal hypertension and abdominal compartment syndrome. Am. J. Kidney Dis. 2011; 57: 159-69.

12. Hoste EA, Cruz DN, Davenport A et al. The epidemiology of cardiac surgery-associated acute kidney injury. Int. J. Artif. Organs 2008; 31: 158-65.

13. Legrand M, Pirracchio R, Rosa A et al. Incidence, risk factors and prediction of post-operative acute kidney injury following cardiac surgery for active infective endocarditis: An observational study. Crit. Care 2013; 17: R220.

14. Ozcakar ZB, Yalcinkaya F, Altas B et al. Application of the new classification criteria of the Acute Kidney Injury Network: A pilot study in a pediatric population. Pediatr. Nephrol. 2009; 24: 1379-84.

15. Bradley EL 3rd. A clinically based classification system for acute pancreatitis. Summary of the International Symposium on Acute Pancreatitis, Atlanta, Ga, September 11 through 13, 1992. Arch. Surg. 1993; 128: 586-90.

16. Bone RC, Sprung CL, Sibbald WJ. Definitions for sepsis and organ failure. Crit. Care Med. 1992; 20: 724-6.

17. Vincent JL. Clinical sepsis and septic shock - definition, diagnosis and management principles. Langenbecks Arch. Surg. 2008; 393 817-24.

18. Malbrain ML, Cheatham ML, Kirkpatrick A et al. Results from the international conference of experts on intra-abdominal hypertension and abdominal compartment syndrome. I. Intensive Care Med. 2006; 32: 1722-32.

19. Mortele KJ, Wiesner W, Intriere L et al. A modified CT severity index for evaluating acute pancreatitis: Improved correlation with patient outcome. AJR Am. J. Roentgenol. 2004; 183: 1261-1265.

20. Ranson JH, Rifkind KM, Roses DF et al. Prognostic signs and the role of operative management in acute pancreatitis. Surg. Gynecol. Obstet. 1974; 139: 69-81.

21. Knaus WA, Draper EA, Wagner DP et al. Prognosis in acute organ-system failure. Ann. Surg. 1985; 202: 685-93.

22. Chang RWS, Jacobs S, Lee B. Gastrointestinal dysfunction among intensive care unit patients. Crit. Care Med. 1987; 15: 909-14.

23. Wilmer A. ICU management of severe acute pancreatitis. Eur. J. Intern. Med. 2004; 15: 274-80.
24. Lin HY, Lai JI, Lai YC, Lin PC, Chang SC, Tang GJ. Acute renal failure in severe pancreatitis: A population-based study. Ups. J. Med. Sci. 2011; 116: 155-9.

25. Li H, Qian Z, Liu Z, Liu X, Han X, Kang H. Risk factors and outcome of acute renal failure in patients with severe acute pancreatitis. J. Crit. Care 2010; 25: 225-9.

26. Tran DD, Oe PL, de Fijter CW, van der Meulen J, Cuesta MA Acute renal failure in patients with acute pancreatitis: Prevalence, risk factors, and outcome. Nephrol. Dial. Transplant. 1993; 8: 1079-84

27. Bagshaw SM, Uchino S, Bellomo R et al. Septic acute kidney injury in critically ill patients: Clinical characteristics and outcomes. Clinical journal of the American Society of Nephrology. Clin. J. Am Soc. Nephrol. 2007; 2: 431-9.

28. Ricci Z, Cruz D, Ronco C. The RIFLE criteria and mortality in acute kidney injury: A systematic review. Kidney Int. 2008; 73 538-46.

29. Mehta RL, Pascual MT, Gruta CG, Zhuang S, Chertow GM. Refining predictive models in critically ill patients with acute renal failure. J. Am. Soc. Nephrol. 2002; 13: 1350-57.

30. Company L, Saez J, Martinez J et al. Factors predicting mortality in severe acute pancreatitis. Pancreatology 2003; 3: 144-8.

31. Ljutic D, PiplovicVukovic T, Raos V, Andrews P. Acute renal failure as a complication of acute pancreatitis. Ren. Fail. 1996; 18: 629-33.

32. Chiutu L, Georgescu E, Purcaru F, Nemes R, Georgescu I. Severity factors of acute renal failure in severe acute pancreatitis. Chirurgia (Bucur) 2006; 101: 609-13.

33. Hoste EA, Lameire NH, Vanholder RC, Benoit DD, Decruyenaere JM, Colardyn FA. Acute renal failure in patients with sepsis in a surgical ICU: Predictive factors, incidence, comorbidity, and outcome. J. Am. Soc. Nephrol. 2003; 14: 1022-30.

34. Uchino S, Kellum JA, Bellomo R et al. Acute renal failure in critically ill patients: A multinational, multicenter study. JAMA 2005; 294: 813-8.

35. Larvin M, McMahon MJ. APACHE-II score for assessment and monitoring of acute pancreatitis. Lancet 1989; 2: 201-5.

36. Wilson C, Heath DI, Imrie CW. Prediction of outcome in acute pancreatitis: A comparative study of APACHE II, clinical assessment and multiple factor scoring systems. Br. J. Surg. 1990; 77: 1260-64.

37. Vincent JL, Moreno R, Takala J et al. The SOFA (Sepsis-related Organ Failure Assessment) score to describe organ dysfunction/failure. On behalf of the Working Group on Sepsis-Related Problems of the European Society of Intensive Care Medicine. Intensive Care Med. 1996; 22: 707-10.

38. Balthazar EJ, Robinson DL, Megibow AJ, Ranson JH. Acute pancreatitis: Value of CT in establishing prognosis. Radiology 1990; 174: 331-6.

39. Gurleyik G, Emir S, Kilicoglu G, Arman A, Saglam A. Computed tomography severity index, APACHE II score, and serum CRP concentration for predicting the severity of acute pancreatitis. JOP 2005; 6: 562-7. 